\title{
CONCEPTUAL ANALYSIS OF SUSTAINABLE DEVELOPMENT \\ AND SUSTAINABLE DEVELOPMENT
}

\section{ANALISIS CONCEPTUAL DEL DESARROLLO SOSTENIBLE Y EL DESARROLLO SUSTENTABLE}

Recibido: 15 de enero 2014- aceptado: 26 de abril 2014

\author{
Efraín De La Hoz Granadillo \\ Universidad de Cartagena \\ Ludys López Polo² \\ Universidad Simón Bolívar
}

\begin{abstract}
Keywords:
Sustainable Development, Sustainable Development, Environmental

Management,Development
\end{abstract}

\section{Abstract}

This article is a reflection on the concepts of sustainable development and sustainable development terms that generate discussion on its meaning in the field of environmental management. For which a review of the related literature developed for the purpose of this discussion. As a result of this review it was established conceptual aspects to analyze more deeply the concept of sustainable development and sustainable development which allowed determining matching conditions, but also of divergence which can give better items so plans are developed development in the context of environmental management.

\begin{tabular}{|c|c|}
\hline labra & \\
\hline Gestión & Ambiental, \\
\hline Desarrollo & Sostenible, \\
\hline $\begin{array}{l}\text { Desarrollo } \\
\text { Desarrollo. }\end{array}$ & Sustentable, \\
\hline
\end{tabular}

1 Docente Asistente Programa Administración Industrial. Universidad de Cartagena. Doctorante Programa Doctoral en Ciencias Sociales. Universidad del Zulia. edelahozg@unicartagena.edu.co

2. Docente de planta Programa Ingeniería Industrial. Universidad Simón Bolívar. Doctorante Programa Doctoral en Ciencias Sociales. Universidad del Zulia. lulopez@unisimonbolivar.edu.co

*Este artículo es asociado al proyecto: Análisis Conceptual Del Desarrollo Sostenible Y El Desarrollo Sustentable 


\section{INTRODUCCIÓN}

Conceptualmente, el medio ambiente es un sistema y constituye todos aquellos elementos del entorno con los cuales se interactúa y en el que se puede ejercer un impacto negativo o positivo, $Y$ en el que el hombre desarrolla sus actividades en procura de obtener mejores condiciones de vida. En este sentido la gestión ambiental se convierte en una herramienta importante en la canalización de iniciativas y acciones que permitan generar desarrollo económico, productivo y social a la vez que se logren mantener y mejorar las condiciones del medio ambiente.

La gestión ambiental y el cuidado del medio ambiente no sólo es un compromiso de las naciones ante el cambio climático, sino que debe ser una condición de conciencia y responsabilidad tanto de las organizaciones productivas como de cada miembro de la sociedad. De manera que el establecimiento y mejoramiento de la calidad de vida debe ir de la mano con el mejoramiento de la calidad del medio ambiente y su sostenibilidad.

Por tanto, el desarrollo de las naciones debe ser integral de manera que se articulen variables económicas, tecnológicas, productivas, sociales, culturales, ambientales, etc. En este sentido el desarrollo se concibe como una condición en el que los países logran alcanzar el equilibrio que les permita de manera continua mantener o mejorar las condiciones de vida tanto de las generaciones presentes como futuras.

\section{RESULTADOS}

\section{A. GESTIÓN AMBIENTAL}

La gestión ambiental es un tema que en las últimas décadas ha tenido especial interés dado el cambio climático y el efecto que tienen las regulaciones de este tema frente a las actividades económicas, sociales y culturales. En este sentido es conveniente definir el medio ambiente como un sistema en el que interactúan diferentes elementos físicos, biológicos, sociales, culturales y estéticos y que constituyen el entorno donde se desarrolla la vida. [1].

Según Ortega \& Rodríguez [2] la gestión ambiental es el conjunto de acciones encaminadas a mantener el capital ambiental de manera que se logre elevar el patrimonio natural y la calidad de vida de las personas, teniendo en cuenta las restricciones impuestas por las relaciones económicas y sociales que condicionan este objetivo. De esta manera la gestión ambiental constituye la asignación de recursos materiales, económicos y sociales necesarios para lograr estándares o niveles de calidad ambiental atendiendo a la conservación de los ecosistemas y las relaciones ecológicas que presentan entre ellos [3].
Por su parte Granero \& Ferrando [4] establecen que el objetivo de la Gestión ambiental es el incremento de los niveles de calidad para lo cual se deben tomar las acciones necesarias que impidan la degradación y contaminación del medio ambiente, así como aquellas orientadas a recuperar y sanear el entorno, devolviéndole su capacidad de respuesta.

\section{B. CONCEPTO DE DESARROLLO SOSTENIBLE}

Desde la década comprendida entre los años 40 y los 70 en la cual la sociedad disponía de buenos niveles de vida, comenzó la preocupación de los gobiernos, las organizaciones y la sociedad en general por el tema ambiental y el desarrollo sostenible como alternativa para abordar la necesidad de crecimiento económico al mismo tiempo que se logre un desarrollo social real, y mantener el equilibrio ecológico, el establecimiento de normas que frenen el impacto de la industrialización, así como la disyuntiva entre si el mejoramiento de la situación social y ambiental deben ser iniciativa de los gobiernos o de la empresa privada [5]. Sin embargo, han pasado más de ocho décadas y el problema del desarrollo sostenible hoy más que nunca ha cobrado vigencia y preocupación dado los impactos que se están observando en el medio ambiente dado el acelerado desarrollo e industrialización de la sociedad, con lo cual se hace el cuestionamiento en el sentido si el desarrollo sostenible es alcanzable.

Por su parte, Moller [6]) ubica el desarrollo sostenible como elemento central en el ámbito político, académico y en la discusión de proyectos de desarrollo pero que al momento de canalizar su implementación se sigue malinterpretando y contradiciendo las estrategias para alcanzarlo. Propone tres objetivos fundamentales con el fin de clarificar el concepto de desarrollo sostenible, los cuales propenden por garantizar la existencia del género humano, mantener los niveles productivos de la sociedad e identificar alternativas de desarrollo de sociedad. Así mismo, establece principios para el desarrollo sostenible con el objeto de garantizar la salud de las personas, asegurar la existencia de las personas, por medio del uso sostenible de los recursos naturales no renovables. En este sentido, es importante el uso sostenido del medio ambiente que permita generar un equilibrio en el largo plazo a partir del desarrollo sostenible del capital material, el recurso huno y el conocimiento humano. Lo cual debe permitir la igualdad de oportunidades de las personas y protección de la diversidad cultural, el equilibrio entre las diferencias entre ricos y pobres, para lo cual es necesario garantizar los recursos y las clases sociales.

James, S. y Lathi, T. [7], han establecido principios de sostenibilidad que implican la reducción en la utilización 
de los combustibles fósiles y la explotación de las minas de metales y minerales, la dependencia de materiales, químicos sintéticos y de otras sustancias no naturales, disminuir la irrupción a la naturaleza y su degradación y satisfacer las necesidades humanas de una manera justa y eficiente. Por su parte autores como Anastas y Eghbali [8], proponen principios de química verde o química sostenible, orientados a la prevención en la generación de residuos, síntesis químicas menos peligrosas, la eficiencia energética, utilización de materia prima renovable, fabricación de productos biodegradables entre otros.

\section{CONCEPTO DE DESARROLLO SUSTENTABLE}

El termino desarrollo sustentable fue utilizado por primera vez por la primera ministra Noruega Gro Harlem Brundtland quien lo define en el documento "Nuestro Futuro Común", como la satisfacción de las necesidades del presente sin poner en riesgo la posibilidad de que generaciones venideras puedan satisfacer sus propias necesidades [9]. La anterior definición es amplia y no deja claro cuáles son los principios bajo los cuales se establece la noción de equidad para la satisfacción de las necesidades del presente, así como el cuidado que se le debe dar al medio ambiente de manera que se garantice que no se deteriore la satisfacción de las necesidades de las generaciones futuras.

Así mismo, organizaciones internacionales como la Organización de las Naciones Unidas (ONU), La Organización para la Cooperación y el Desarrollo Económico, el Banco Mundial, entre otros, utilizan de manera frecuente el término de desarrollo sustentable, que constituye tres elementos básicos como son; sustentabilidad ecológica que involucra la conservación y el cuidado del medio ambiente y los seres vivos, sustentabilidad social la cual pone de manifiesto la necesidad de disponer mejores condiciones de vida para el desarrollo del ser humano y la sustentabilidad económica que supone la explotación de los recursos de la naturaleza. De lo anterior, el desarrollo sustentable se define como un equilibrio armónico entre estas dimensiones de manera que se logre la equidad entre las generaciones actuales y futuras [10].

\section{DESARROLLO SUSTENTABLE VS DESARROLLO SOSTENIBLE}

Autores como Rodríguez y Govea [11] enmarcan el desarrollo sustentable y sostenible como dos corrientes de desarrollo que adoptan caminos diferentes para la utilización de los recursos naturales y la satisfacción de las necesidades del hombre.

Según el rol de la economía el desarrollo sustentable, ofrece un crecimiento económico en el que se explotan los recursos renovables y no renovables con un desarrollo tecnológico que permite equilibrar las desigualdades que se puedan generar en las generaciones futuras. Por el contrario, el desarrollo sostenible ofrece un desarrollo económico y social equilibrado con explotación adecuada de los recursos naturales, propicia un desarrollo ecoambiental, socio-ambiental y sociocultural.

De acuerdo a la concepción de la naturaleza, el enfoque de desarrollo sustentable propende por la explotación de todos los recursos amparado en que la tecnología resolvería los problemas de escasez del futuro, por su parte el enfoque sostenible incorpora la gestión y la protección del medio ambiente, la biodiversidad y la reapropiación sociocultural de la naturaleza.

En lo relacionado con el papel de la tecnología, el enfoque sustentable propone soluciones técnicas mixtas para revertir los efectos sobre el medioambiente, en contraste con el enfoque sostenible que incorpora tecnologías limpias y condiciones tecnológicas que no deterioren el medio ambiente.

A nivel político, el enfoque sustentable acoge ciertas restructuraciones de las instituciones y modificaciones de normas ambientales con un sentido proteccionista, a su vez el desarrollo sostenible incorpora la democratización con una gestión participativa a nivel económico, político, cultural, social y gerencial con control social.

A nivel ideológico, la doctrina del desarrollo sustentable es antropocéntrica ubicando el hombre como factor central del desarrollo, dejando de lado las consideraciones ambientales para la implementación de la tecnología. Por su parte, el enfoque sostenible posibilita la construcción de una conciencia cívica, la conducta ética y el conocimiento proactivo.

En general el desarrollo sustentable promueve el crecimiento económico sin altos niveles de conservación ambiental. El desarrollo sostenible genera responsabilidad social compartida, empoderamiento ciudadano, el desarrollo de valores éticos y compromisos con la naturaleza.

Por otro lado, Reyes-Sánchez, [12] reconocen diferencias entre el desarrollo sostenible y el sustentable, establecen que el concepto dado por la ONU como "sustainable development" corresponde al desarrollo sostenible y debe ser estudiado por los docentes para transmitirlo a la ciudadanía. En Venezuela, Rodríguez y Govea [11] aceptan que el desarrollo sostenible y el sustentable son antropocéntricos pero asumen enfoques con corrientes distintas: El primero es integral con valores, tecnologías limpias y leyes de protección que proporciona bienestar tanto a las generaciones presentes y futuras con cuidado 
ambiental, social y económico. El desarrollo sustentable por el contrario, permite el deterioro del medio ambiente y el establecimiento de brechas en lo social y lo económico, generando diferencias profundas en países desarrollados y subdesarrollados. Lo que a la larga conduce a una crisis global.

\section{CONCLUSIONES}

Tanto el desarrollo sostenible como el desarrollo sustentable hacen uso de recursos del medio ambiente y generan impacto sobre éste, en el propósito de crear condiciones que permitan mejorar la calidad de vida de la humanidad.

Por su parte, el desarrollo sostenible incorpora la variable tiempo en la función de desarrollo de manera que busca generar el bienestar de la generación presente, pero garantizando el dejar a las generaciones futuras condiciones ambientales mejores o por lo menos iguales a las que se tienen en el presente. En este sentido el desarrollo sostenible es socialmente responsable y constituye una concepción de desarrollo en el que se le dan a los recursos un uso ético y equilibrado.

El desarrollo sostenible implica un cambio en las organizaciones y por ende un cambio en las personas que las conforman, finalmente son los miembros de las organizaciones quien tienen la capacidad de pensar y de reorientar los procesos y las actividades que se ejecutan al interior de estas, en este sentido cobra mucha importancia la gestión del conocimiento como herramienta fundamental para que las personas y las organizaciones en general conozcan su entorno y a través de esto establezcan relaciones amigables con el medio, relaciones en la que es necesaria la coordinación de varios actores con el fin de que el desarrollo sostenible se lleve a cabo de una forma integral.

Por el contrario el desarrollo sustentable solo tiene en cuenta el mejoramiento de las condiciones de vida de las generaciones actuales a un nivel que las dificultades generadas por la explotación de los recursos no renovables, así como el deterioro o degradación de la naturaleza puedan ser superadas por las generaciones futuras con el desarrollo de la ciencia y la tecnología.

Por su parte, Leff [13] acepta que existen diferencias entre desarrollo sostenible y sustentable pero señala que ambas ocultan la real crisis ambiental y ecológica derivada de la industria, considera al modelo capitalista como degradador del ambiente, de un sistema social agresor del medio ambiente, es utilitarista en el sentido de su bienestar egoísta sin importar el de los demás, falta del sentido de nosotros.
De lo anterior se evidencia la necesidad que las naciones, organizaciones y la sociedad en general propendan por incorporar modelos de desarrollo amigables con el medio ambiente [14].

\section{REFERENCIAS}

[1] D. Gómez, Evaluación de impacto ambiental. Editorial Grupo Mundi- Prensa. Segunda edición. ISBN: 84-8476084-7 Madrid- España. Pp. 37, 2002.

[2] R. Ortega, \& I. Rodríguez, Manual de Gestión del Medio Ambiente. Fundación M.A.P.F.R.E. Madrid. 1994.

[3] V. Fernández, Los instrumentos de la gestión ambiental en la empresa. Ediciones Mundi- Prensa. Madrid-España. ISBN: 84-7114-648-7. Pp. 60. 1996.

[5] E. Urteaga, El debate Internacional sobre el desarrollo sostenible. Investigaciones geográficas. Núm. 46: 127 137. 2008.

[6] R. Moller, Principios del desarrollo sostenible para América Latina. Ingeniería de recursos naturales y del ambiente. Núm. 9. 101 - 110. 2010.

[7] S. James, y T. Lathi, Eco-municipalities: Sweden and the United States: A Systems Approach to Creating Communities (en

línea),. http://www.knowledgetemplates.com/sja/ecom unic.htm. Acceso: 22 de junio 2013. 2003.

[8] P. Anastas, \& N. Eghbali, Green Chemistry: Principles and Practice. 2010. Chemical Society Reviews. (base de datos en línea), 39, 301-312. http://www.bidi.uam.mx. Acceso: 17 de enero 2013.

[9] D. Fernández, El principio de integración del medio ambiente en la Unión Europea. Revista Cuaderno de Realidades Sociales. No. 55/56. España, pp23 - 44. 2000. [10] R. Sánchez, La transición hacia el desarrollo sustentable: perspectivas de América Latina y el Caribe. Capítulo 13: Sustentabilidad Urbana, Descentralización y Gestión Local. Edita Secretaría del Medio Ambiente y Recursos Naturales, Instituto Nacional de ecología, Universidad Autónoma Metropolitana, Organización de las Naciones Unidas, Programa de las Naciones Unidas para el Medio Ambiente. Pp. 311. México. 2002.

[11] I. Rodríguez, \& H. Govea, El discurso del desarrollo sustentable en América Latina. 2006. Revista Venezolana de Economía y Ciencias Sociales, 12(2) 37-63. Recuperado de http://www.redalyc.org/articulo.oa?id=17712202

[12] L. B. Reyes-Sánchez, Aportación de la química verde a la construcción de una ciencia socialmente responsable, Educ. quím.: 23(2), 222-229. 2012.

[13] E. G. Leff, La geopolítica de la biodiversidad y el desarrollo sustentable: Economización del mundo, racionalidad ambiental y reapropiación social de la naturaleza. 2003. Revista Líder: 11, 21-37. Osorno: Universidad de los Lagos. 
[14] Vértice Editorial. Gestión medioambiental: conceptos básicos. Publicaciones Vértice. España. ISBN: 978-84-

9931-129-6. Pp.2.2010. 